\title{
Isolation of Novel Vincristine and Vinblastine Producing Streptomyces Species from Catharanthus Roseus Rhizospheric Soil
}

Onja Herivony Andriambeloson ( $\sim$ herionja@ymail.com)

Centre National de Recherches sur l'Environnement https://orcid.org/0000-0003-0660-8255

Rabenandrasana Mamitiana Alain Noah

Institut Pasteur de Madagascar

Andrianantenaina Rigobert

Centre National de Recherches sur l'Environnemnr

Collard Jean-Marc

Center for microbes, development and health, Institut Pasteur Shanghai/chinese

Ramaroson Luciano

Centre National de Recherches Météorologiques: Centre National de Recherches Meteorologiques

Rasolomampianina Rado

Centre National de Recherches Météorologiques: Centre National de Recherches Meteorologiques

\section{Research Article}

Keywords: actinomycetes, endophytes, rhizospheric soil, alkaloids, vincristine, vinblastine

Posted Date: November 19th, 2021

DOI: https://doi.org/10.21203/rs.3.rs-1082130/v1

License: (c) (i) This work is licensed under a Creative Commons Attribution 4.0 International License. Read Full License 


\section{Abstract}

Microorganisms could be used as efficient tools to protect high value therapeutic plants against overexploitation and climate change. This work aimed to isolate alkaloids producing endophytic and rhizospheric soil actinomycetes and fungi of Catharanthus roseus. From a total of eleven actinomycetes and eight fungi strains isolated by dilution and plate methods, six telluric actinomycetes were revealed to produce alkaloids according to the precipitation test of their extracts. Revelation by Thin Layer Chromatography method using vinblastine and vincristine standards on the basis of frontal reference values showed that the strain SC8 and the vinblastine, the strain SC7 and the vincristine displayed the same frontal references $(0,89$ and 0,88 , respectively). Whole genome sequencing method showed that both strains belong to the genus Streptomyces with novel species.Moreover, metabolic pathways analysis from their genomes allowed to detect three enzymes involved in the biosynthesis of terpenoid backbone leading to that of terpenoid indole alkaloids.

\section{Introduction}

Disappearance threatof certain high therapeutic value plants is a significant and non-negligible problem that has to be solved, view their importance in medicines production as well as their impact in diseases treatment. According to the Kew Gardens Botanical Research centerin 2016, one out of five plant species is threatened of disappearance including medicinal plants. Some of them are reported to be disappeared at a slow rhythm;overexploitation and climate change could be the probable causes. In 2004, the World Health Organization (WHO) inventoried Catharanthus roseus (Madagascar periwinkle) among endangered medicinal plants. It is an endemic plant of Madagascar traditionally used for its "appetite-suppressant" property, its ability to heal wasp stings and its curative property as wormer, wounds disinfectant, antimalarial and diuretic in infusion. However, the antitumor property of the plant was highlighted between 1958 and 1965. Catharanthus roseus has, then, been used to treat certain cancerous diseases. The plant produces several alkaloids, among which two are well-known for their anticancer properties and as the second best anticancer drugs used in the world (Hadagali et al. 2017): the vinblastine particularly used to treat Hodking's disease and the vincristine, for children's acute leukemia treatment. These two molecules are nitrogenous heterocycles of alkaloids family and the processes of their extraction revealed that a ton of whole periwinkle plant provides $1 \mathrm{~g}$ of vinblastine and $10 \mathrm{~g}$ of vincristine. Indeed, the extraction ofbioactive molecules is the main causeof disappearance of certain medicinal plant species. Several strategies are now adopted to protect endangered plants: in-situ conservation (for protection of natural area by creating parks or reserves), ex-situ conservation (creation of seeds or living plants bank), culture intensification and domestication of the most exploited plants. For research side, the works are focused to cultivars development, active principle synthesis and recently cloning and genetic modification (GMO). Besides, this work allows to demonstrate that microbiology is a promising and effective tool to produce bioactive molecules comparable to those of the plant for its preservation. Many research works demonstrated that endophytic microorganisms such as actinomycetes and fungi exhibited the same biological activities (Akshatha et al. 2014; Herivony et al. 2016) and produced the same secondary metabolites as the host-plants(Shweta et al. 2010; Amna et al. 2006; Caruso et al. 2000; Zhao et al. 2013). Some works reported also that actinomycete from rhizospheric soil of medicinal plant displayed the same biological activities than the plant (Herivony et al. 2016). Then, the objective of this reported work was to isolate vinblastine and vincristine producing actinomycetes and fungi from Catharanthusroseus and its rhizospheric soil. Cultural, morphological and molecular characterizations of the target isolates were established in this study.

\section{Materials And Methods}

\subsection{Plant and soil sampling}

Catharanthus roseus whole plant and its rhizospheric soil were collected from decorative plants garden of National Center of Environmental Research, Antananarivo-Madagascar. Rhizospheric soil was sampled at $10 \mathrm{~cm}$ of depth under the plant at the harvest moment in April 2018. Plant sample was, then, put onto kraft paper and soil sample into sterile plastic bag; they were 
transported immediately to the laboratory for actinomycetes and fungi isolation (plant) and for drying in a dessicator during one week (soil rhizospheric).

\subsection{Isolation of actinomycetes and fungi strains 2.2.1. Isolation of endophytic strains}

Endophytes isolation from different organs of Catharanthus roseus (flowers, leaves, stem, fruits and roots) was performed using plate method (Andriambeloson et al. 2014) on Starch Casein Agar (SCA) for actinomycetes and on Potato Dextrose Agar (PDA) for fungi.

Vegetative organs were washed with tap water and soaked in the ethanol $70 \%$ for 30 s, then in the sodium hypochloride (3\%) for $3 \mathrm{~min}$, followed by three successive washings with distilled water to sterilize their surfaces. Plant organs were then cut into small pieces $(1 \mathrm{~cm})$ and put, firstly onto SCA petri plates supplemented with nystatin $(25 \mathrm{mg} / \mathrm{ml})$, cycloheximide $(50 \mathrm{mg} / \mathrm{ml})$ and nalidixic acid $(25 \mathrm{mg} / \mathrm{ml})$ to suppress fungal and negative bacteria development respectively. Plates were incubated at $30^{\circ} \mathrm{C}$ for one week to one month. On the other hand, plant organs pieces were put onto PDA petri plates supplemented with chloramphenicol $(50 \mathrm{mg} / \mathrm{ml})$ to inhibit bacteria growth. Cultures were incubated at $28^{\circ} \mathrm{C}$ for 5 days.

\subsubsection{Isolation of telluric strains}

Telluric strains isolation was conducted using dilution and heat treatment methods (Oskay et al. 2004) with the same media as cited above for actinomycetes and fungi.

For dilution method, $1 \mathrm{~g}$ of soil sample was suspended in $9 \mathrm{ml}$ of distilled water. Soil suspension was then homogenized on a shaker for $5 \mathrm{~min}$ and a serial dilution from $10^{-1}$ to $10^{-3}$ was prepared. Thereafter, $100 \mu$ l of the dilutions $10^{-2}$ and $10^{-3}$ were put and spread onto SCA and PDA plates. Plates were incubated for one week to one month for actinomycetes growth and for 5 days for fungi development.

For heat treatment method, $1 \mathrm{~g}$ of soil sample was exposed to $100^{\circ} \mathrm{C}$ for $1 \mathrm{~h}$; then suspended in distilled water. The same technique as described above was applied for actinomycetes and fungi isolation.

To differentiate the two strains, actinomycetes colony was characterized by solid colony embedded in the agar medium with vegetative and aerial mycelia (Shriling and Gottlieb 1966). Fungal colony is, however, recognizable by the development of mycelium on the culture media (Alexopoulos and Mims 1979).

\subsection{Purification and conservation of the isolates}

For each isolate, colony was purified by two or three successive subcultures on SCA or PDA media without antibiotic or antifungal. Pure isolates obtained were conserved according to two methods: the agar slants preservation for short-term preservation and the cryoconservation for long-term preservation.

Concerning short-time preservation, the colonies of both strains (actinomycetes and fungi) were subcultured on SCA and PDA slants agar. The cultures were incubated at $30^{\circ} \mathrm{C}$ and $28^{\circ} \mathrm{C}$ for 7 days and 5 days, respectively and stored at $+4^{\circ} \mathrm{C}$ for one year.

For long-time preservation, actinomycetes colonies were cultured on Starch Casein broth with shaking at 150rpm for 7 days at $30^{\circ} \mathrm{C}$. One milliliter $(1 \mathrm{ml})$ of the culture was then poured into cryotubes containing $1 \mathrm{ml}$ of glycerol $(20 \%)$. The mixture was homogenized using vortex and the cryotubes were stored at $-80^{\circ} \mathrm{C}$ for ten years (Isik et al. 1999).

The conservation of fungal strain was performed by refrigerating at +1 to $+10^{\circ} \mathrm{C}$ the minitubes containing sterile distilled water and a small mycelial block agar during about 7 years (Nguyen 2007).

\subsection{Alkaloids test}




\subsubsection{Extracts preparation}

Pure actinomycetes and fungi strains were subcultured in large Petri plates (150mm) containing ISP2 agar (International Streptomyces Project 2) and PDA, respectively. After 8 days of incubation for actinomycetes and 14 days for fungi, secondary metabolites were extracted with ethanol $90^{\circ}$ by maceration for $2 \mathrm{~h}$. The solutions obtained were, then, filtrated with Millipore filter $(0,22 \mu \mathrm{m})$ and the filtrates were dried using speedvac to form the extracts.

\subsubsection{Alkaloids screening}

Extracts were screened for alkaloids presence using Fong et als method (1977) by precipitation test with Dragendorff, Mayer and Wagner reagents. Dried extract was macerated in $3 \mathrm{ml}$ of $\mathrm{H} \mathrm{Cl}(2 \mathrm{~N})$ and the obtained solution was divided in three aliquots in three tubes. Each aliquot was treated with each precipitation reagent and positive result was confirmed if each reagent gave a precipitate.

\subsubsection{Alkaloids extraction}

Alkaloids were extracted from positive extracts using modified dichloromethane (DCM) method (Bouzidi et al. 2011), divided into two steps. The first step was a solid-liquid extraction, $300 \mathrm{mg}$ of extracts and $100 \mathrm{ml}$ of water were mixed in a recipient. Sodium bicarbonate was, then, added until obtaining a $\mathrm{pH}$ 9; the mixture was boiled for $2 \mathrm{~h}$ and cooled at the end of the decoction.

The second step was a liquid-liquid extraction, for which total alkaloids were extracted by DCM. For that, the mixture obtained previously and $50 \mathrm{ml}$ of DCM were put in a separating funnel. The mixture was, then, shaken vigorously and decanted; organic phase was recuperated and evaporated, the dried residue forms the total alkaloids.

\subsection{Vinblastine/Vincristine detection by Thin Layer Chromatography (TLC)}

TLC method was adopted to detect vinblastine and vincristine from the extracted alkaloids. Solvent system used was dichloromethane/methanol/water (8:2:2, v/v/v), the vinblastine (RMPL-PHARMA LLP, India) and the vincristine (CELON Labs, India) were used as standards and the Dragendorff reagent as a revelator. Brown spot on yellow background revealed alkaloids presence. Vinblastine or vincristine molecules in the alkaloid extracts were detected by comparing the frontal references (Rf) of the standards and the extracts which were calculated from the following formula: $\mathrm{Rf}=$ distance of solute/distance of solvent

\subsection{Cultural and morphological characteristics of vinblastine and vincristine producing strains}

Cultural characteristics of the target strains were observed macroscopically on SCA medium by mentioning colonies aspects, the growth, the development of substrate and aerial mycelia and the presence of diffusible pigments other than melanoid pigments after 7,14 and 21 days of incubation at $30^{\circ} \mathrm{C}$.

Morphological characteristics of the target strains were, however, revealed using culture on coverslip technique (Cross 1989). A sterile coverslip was inserted into ISP2 medium plate by forming an angle of $45^{\circ}$. A drop of the inoculum was then put against the coverslip in contact with the medium. After 14 days of incubation at $30^{\circ} \mathrm{C}$, the coverslip was carefully removed from the medium, put on a slide and observed under microscope at the magnification 40x. The morphology of spores, substrate and aerial mycelia was noted.

\subsection{Molecular characterization of vinblastine and vincristine producing strains}


This study was carried out to identify the target isolated strains using whole genome sequencing (Institut Pasteur, Madagascar). The DNAs of pure isolates were extracted from $72 \mathrm{~h}$ old cultures with Qiagen Genomic DNA isolation kit (Qiagen) according to manufacturer's instructions. The purity and the amount of DNA extracted were then assessed with a spectrophotometer Nanodrop (Thermo Fisher Scientific, Waltham, USA). Extracted DNAs were thereafter sequenced by preparing DNA sequencing libraries with the Nextera XT kit (Illumina) and 2×150 bps paired-end sequencing was performed using alllumina NextSeq500 instrument. Sequenced reads were trimmed and clipped using Alien Trimmer (Criscuolo and Brisse 2013) and sequencing errors were corrected using Musket (Liu et al. 2013). De novo assembly was carried out using SPAdes(Bankevich 2012), gene prediction and annotation were performed using PATRIC (Pathosystems Resource Integration Center) (James et al. 2020). Whole-genome-based taxonomic analysis was performed by Type Strain Genome Server (TYGS) (Meier-Kolthoff and Göker 2019).The average nucleotide identity (ANI) and genome-to-genome distances (GGDs)/ digital DNA-DNA hybridization (dDDH) values were calculated in online calculator (http://enve-omics.ce.gatech.edu/g-matrix/index) (Rodriguez and Konstantinidis 2016) and Genome-to-Genome Distance Calculator (GGDC; http://ggdc.dsmz.de) (MeierKolthoff et al. 2013), respectively, with closest strains identified with TYGS. The phylogenomic tree was constructed using FastME (Lefort et al. 2015) from the genome blast distance phylogeny (GBDP). The trees were rooted at the midpoint and the branch supports were inferred from 100 pseudo-bootstrap replicates. Furthermore, a phylogenic tree based on 16S was established in order to confirm the taxonomy of the strains.

\subsubsection{Specialty genes}

Besides the identification of the target isolates, the genes encoding enzymes implied in the biosynthesis of secondary metabolites, particularly the two anticancers produced by the target isolates were assessed from the analysis of metabolic pathways with PATRIC to verify the work hypothesis.

\section{Results}

\subsection{Actinomycetes and fungi isolation}

After five days and one week of incubation, fungi and actinomycetes were identified, respectively, according to their own macroscopic aspect. Actinomycetes were characterized by hard colonies implanted in the agar while fungi were easily known by their mycelia development on the culture medium.

\subsubsection{Endophytic actinomycetes and fungi}

Isolation of actinomycetes and fungi from different organs of Catharanthus roseus using plate method showed that only, one actinomycete strain was obtained from the stem of the plant. Though, six fungi were isolated from the plant of which one fungus from the flowers, one fungus from the leaves, two from the stem and two from the roots. Endophytes isolated were coded EA (actinomycete) and EF1 to EF6 (fungi).

\subsubsection{Telluric actinomycetes and fungi}

Dilution and heat treatment methods allowed the isolation of ten actinomycetes strains and two fungi strains from Catharanthus roseus rhizospheric soil. The repartition of actinomycetes and fungi strains from Catharanthus roseus plant and its rhizospheric soil is shown in the figure 1. Telluric strains isolated were coded SC1 to SC10 (actinomycetes), SF1 and SF2 (fungi).

\subsection{Alkaloids screening}

For Endophytes, according to the results obtained, neither actinomycete extracts nor fungal extracts contained alkaloids. Six telluric actinomycetes extracts showed, however, positive results for alkaloids test (SC3, SC4, SC5, SC6, SC7 and SC8). None of the two rhizospheric soil fungi extracts contained alkaloids. Their yield varied from $2,3 \%$ to $4,6 \%$ (Table 1 ) and the strain sc6 produced more alkaloids. 
Table 1

Alkaloids yield from actinomycetes extracts

\begin{tabular}{|lllllll|}
\hline Actinomycetesextracts & SC3 & SC4 & SC5 & SC6 & SC7 & SC8 \\
\hline Rawextractweight (mg) & 300 & & & & & \\
\hline Alkaloidsweight (mg) & 7 & 10 & 11 & 14 & 9 & 11 \\
\hline Alkaloidsyield (\%) & $\mathbf{2 , 3}$ & $\mathbf{3 , 3}$ & $\mathbf{3 , 6}$ & $\mathbf{4 , 6}$ & $\mathbf{3}$ & $\mathbf{3 , 6}$ \\
\hline
\end{tabular}

\subsection{Vinblastine/vincristine revelation}

The results of TLC method showed that the extract from SC8 strain possessed the same frontal reference as vinblastine and the extract from SC7 had the same frontal reference as vincristine (figure 2, Table 2). Thus, these two strains produced the two target alkaloids.

Table 2

Frontal references of alkaloids extracts from telluric actinomycetes

\begin{tabular}{|ll|}
\hline Alkaloids extracts & Rf $^{\mathbf{a}}$ \\
\hline VBL $^{\mathrm{b}}$ & $\mathbf{0 , 8 9}$ \\
VCR $^{\mathrm{C}}$ & $\mathbf{0 , 8 8}$ \\
SC3 & 0,85 \\
SC4 & 0,87 \\
SC5 & 0,86 \\
SC6 & 0,86 \\
SC7 & $\mathbf{0 , 8 8}$ \\
SC8 & $\mathbf{0 , 8 9}$ \\
\hline
\end{tabular}

a: frontal reference; b: vinblastine; c: vincristine,

\subsection{Cultural and morphological aspects of vinblastine and vincristine producing strains}

The strain SC8, a vinblastine producing actinomycete isolated from the rhizospheric soil of Catharanthus roseus on SCA medium showed large size colonies of $4 \mathrm{~mm}$ in diameter, opaque, hunchbacked with notched edge. The colonies showed creamy aerial and substrate mycelia, the strain produced yellow diffusible pigment. Microscopically, the strain showed weakly ramified vegetative mycelium; aerial mycelium was less developed with a few isolated spores.

The other strain, SC7 which produced the vincristine have got small and powdery colonies of $2 \mathrm{~mm}$ in diameter with white aerial mycelium and beige substrate mycelium. The strain SC7 produced yellow diffusible pigment. Its microscopic aspect showed branched vegetative mycelium and less developed aerial mycelium with also isolated spores (figure 3).

\subsection{Molecular characterization of the two vinblastine and vincristine producing actinomycetes}

\subsubsection{ANI and DDH values of SC7 and SC8 genome}

Compared to 14 Streptomyces genomes closest to the two target isolates, the highest ANI values were obtained with Streptomyces exfoliatus NRRL B-2924 (90,71\%) and Streptomyces zaomyceticus JCM 4864 (90,21\%) while the lowest values 
were obtained for Streptomyces lateritius JCM 4389 (85,57\%) and Streptomyces omiyaensis JCM 4806 (85,93\%) for the strain SC7. The DDH varied between $33,2 \%$ and $47,9 \%$.

For the strain SC8, the highest ANI values were obtained with Streptomyces exfoliatus NRRL B-2924 (90,72\%) and Streptomyces zaomyceticus JCM 4864(90,24\%), the lowest values were obtained for Streptomyces omiyaensis JCM 4806 (85,96\%) and Streptomyces lateritius JCM 4389 (85,61\%). The DDH varied between 32,7\% and 47,5\% (Table 3).

All ANI values were below the generally accepted species delineation cutoff 95-96\% and corresponding pair wise in silico DNA-DNA hybridization (DDH) values were all below 70\%. The ANI and DDH values between the two strains SC7 and SC8 were $99,97 \%$ and $99,6 \%$, respectively. It indicates that both strains belong to the same species that is identified as a novel species. 
Table 3

ANI and dDDH matrix for the two strains SC7 and SC8 with reference strains in TYGS database

\begin{tabular}{|c|c|c|c|c|c|c|c|c|c|c|}
\hline $\begin{array}{l}\text { Target } \\
\text { isolates }\end{array}$ & $\begin{array}{l}\text { Reference } \\
\text { strains (TYGS } \\
\text { database) }\end{array}$ & $\begin{array}{l}\text { Assembly } \\
\text { accession }\end{array}$ & ANI & $\begin{array}{l}\mathrm{dDDH} \\
(\mathrm{dO}, \\
\text { in \%) }\end{array}$ & $\begin{array}{l}\text { C.I. } \\
\text { (d0, } \\
\text { in \%) }\end{array}$ & $\begin{array}{l}\mathrm{dDDH} \\
(\mathrm{d} 4, \\
\text { in \%) }\end{array}$ & $\begin{array}{l}\text { C.I. } \\
\text { (d4, } \\
\text { in \%) }\end{array}$ & $\begin{array}{l}\text { dDDH } \\
(\mathrm{d} 6, \\
\text { in \%) }\end{array}$ & $\begin{array}{l}\text { C.I. } \\
\text { (d6, } \\
\text { in \%) }\end{array}$ & $\begin{array}{l}\text { G+C } \\
\text { content } \\
\text { difference } \\
\text { (in \%) }\end{array}$ \\
\hline SC7 & $\begin{array}{l}\text { Streptomyces } \\
\text { bikiniensis } \\
\text { NRRL B-1049 }\end{array}$ & GCA_000716465 & 88,74 & 39,4 & $\begin{array}{l}{[36.0} \\
- \\
42.9]\end{array}$ & 34,9 & $\begin{array}{l}{[32.5} \\
- \\
37.4]\end{array}$ & 37,6 & $\begin{array}{l}{[34.6} \\
- \\
40.6]\end{array}$ & 0,89 \\
\hline SC7 & $\begin{array}{l}\text { Streptomyces } \\
\text { bikiniensis } \\
\text { NRRL ISP- } \\
5581\end{array}$ & GCA_000716245 & 88,91 & 34,7 & $\begin{array}{l}{[31.4} \\
- \\
38.3]\end{array}$ & 35,9 & $\begin{array}{l}{[33.5} \\
-38.5]\end{array}$ & 33,8 & $\begin{array}{l}{[30.9} \\
- \\
36.9]\end{array}$ & 0,81 \\
\hline SC7 & $\begin{array}{l}\text { Streptomyces } \\
\text { cinereoruber } \\
\text { JCM } 4205\end{array}$ & GCA_014649095 & 88,78 & 40,1 & $\begin{array}{l}{[36.7} \\
- \\
43.5]\end{array}$ & 34,9 & $\begin{array}{l}{[32.4} \\
-37.4]\end{array}$ & 38,1 & $\begin{array}{l}{[35.2} \\
- \\
41.2]\end{array}$ & 0,88 \\
\hline SC7 & $\begin{array}{l}\text { Streptomyces } \\
\text { exfoliatus } \\
\text { NRRL B-2924 }\end{array}$ & GCF_000725545 & 90,71 & 47,9 & $\begin{array}{l}{[44.5} \\
-51.3]\end{array}$ & 40,2 & $\begin{array}{l}{[37.7} \\
- \\
42.7]\end{array}$ & 46 & $\begin{array}{l}{[43.0} \\
- \\
49.0]\end{array}$ & 0,23 \\
\hline SC7 & $\begin{array}{l}\text { Streptomyces } \\
\text { gardneri JCM } \\
4375\end{array}$ & GCA_014655085 & 88,79 & 40,4 & $\begin{array}{l}{[37.1} \\
- \\
43.9]\end{array}$ & 35,4 & $\begin{array}{l}{[32.9} \\
-37.9]\end{array}$ & 38,5 & $\begin{array}{l}{[35.6} \\
- \\
41.6]\end{array}$ & 0,53 \\
\hline SC7 & $\begin{array}{l}\text { Streptomyces } \\
\text { lateritius JCM } \\
4389\end{array}$ & GCA_014649715 & 85,57 & 33,2 & $\begin{array}{l}{[29.8} \\
- \\
36.7]\end{array}$ & 28,7 & $\begin{array}{l}{[26.4} \\
- \\
31.2]\end{array}$ & 31,1 & $\begin{array}{l}{[28.2} \\
- \\
34.2]\end{array}$ & 0,84 \\
\hline SC7 & $\begin{array}{l}\text { Streptomyces } \\
\text { litmocidini } \\
\text { JCM } 4394\end{array}$ & GCA_014649755 & 88,87 & 40,7 & $\begin{array}{l}{[37.3} \\
- \\
44.2]\end{array}$ & 35,7 & $\begin{array}{l}{[33.3} \\
-38.2]\end{array}$ & 38,8 & $\begin{array}{l}{[35.9} \\
- \\
41.9]\end{array}$ & 0,58 \\
\hline SC7 & $\begin{array}{l}\text { Streptomyce } \\
\text { snarbonensis } \\
\text { JCM } 4147\end{array}$ & GCA_014649015 & 88,79 & 42 & $\begin{array}{l}{[38.6} \\
- \\
45.4]\end{array}$ & 35,1 & $\begin{array}{l}{[32.6} \\
- \\
37.6]\end{array}$ & 39,7 & $\begin{array}{l}{[36.8} \\
- \\
42.8]\end{array}$ & 0,29 \\
\hline SC7 & $\begin{array}{l}\text { Streptomyces } \\
\text { nashvillensis } \\
\text { JCM } 4498\end{array}$ & GCA_014650095 & 89,22 & 41,3 & $\begin{array}{l}{[38.0} \\
- \\
44.8]\end{array}$ & 36,5 & $\begin{array}{l}{[34.1} \\
-39.0]\end{array}$ & 39,6 & $\begin{array}{l}{[36.6} \\
- \\
42.6]\end{array}$ & 0,1 \\
\hline SC7 & $\begin{array}{l}\text { Streptomyces } \\
\text { omiyaensis } \\
\text { JCM } 4806\end{array}$ & GCA_014650895 & 85,93 & 34,8 & $\begin{array}{l}{[31.4} \\
- \\
38.3]\end{array}$ & 29,3 & $\begin{array}{l}{[26.9} \\
- \\
31.8]\end{array}$ & 32,5 & $\begin{array}{l}{[29.5} \\
- \\
35.6]\end{array}$ & 1,69 \\
\hline SC7 & $\begin{array}{l}\text { Streptomyces } \\
\text { tanashiensis } \\
\text { JCM } 4086\end{array}$ & GCA_014648895 & 89,21 & 40,9 & $\begin{array}{l}{[37.6} \\
- \\
44.4]\end{array}$ & 36,4 & $\begin{array}{l}{[34.0} \\
-39.0]\end{array}$ & 39,2 & $\begin{array}{l}{[36.3} \\
- \\
42.3]\end{array}$ & 0,04 \\
\hline SC7 & $\begin{array}{l}\text { Streptomyces } \\
\text { venezuelae } \\
\text { ATCC } 10712\end{array}$ & GCA_000253235 & 89,66 & 46,2 & $\begin{array}{l}{[42.8} \\
-49.6]\end{array}$ & 37,7 & $\begin{array}{l}{[35.3} \\
-40.3]\end{array}$ & 43,9 & $\begin{array}{l}{[40.9} \\
- \\
46.9]\end{array}$ & 0,3 \\
\hline SC7 & $\begin{array}{l}\text { Streptomyces } \\
\text { wedmorensis } \\
\text { NRRL } 3426\end{array}$ & GCF_000716445 & 88,92 & 39,7 & $\begin{array}{l}{[36.3} \\
- \\
43.1]\end{array}$ & 35,6 & $\begin{array}{l}{[33.2} \\
- \\
38.1]\end{array}$ & 38 & $\begin{array}{l}{[35.0} \\
- \\
41.0]\end{array}$ & 0,12 \\
\hline SC7 & $\begin{array}{l}\text { Streptomyces } \\
\text { zaomyceticus } \\
\text { JCM } 4864\end{array}$ & GCA_014656215 & 90,21 & 45,7 & $\begin{array}{l}{[42.3} \\
- \\
49.1]\end{array}$ & 38,8 & $\begin{array}{l}{[36.3} \\
- \\
41.3]\end{array}$ & 43,7 & $\begin{array}{l}{[40.7} \\
- \\
46.8]\end{array}$ & 0,1 \\
\hline SC8 & $\begin{array}{l}\text { Streptomyces } \\
\text { bikiniensis } \\
\text { NRRL B-1049 }\end{array}$ & GCA_000716465 & 88,78 & 39,2 & $\begin{array}{l}{[35.8} \\
- \\
42.7]\end{array}$ & 35 & $\begin{array}{l}{[32.6} \\
- \\
37.5]\end{array}$ & 37,4 & $\begin{array}{l}{[34.5} \\
- \\
40.5]\end{array}$ & 0,91 \\
\hline
\end{tabular}




\begin{tabular}{|c|c|c|c|c|c|c|c|c|c|c|}
\hline $\begin{array}{l}\text { Target } \\
\text { isolates }\end{array}$ & $\begin{array}{l}\text { Reference } \\
\text { strains (TYGS } \\
\text { database) }\end{array}$ & $\begin{array}{l}\text { Assembly } \\
\text { accession }\end{array}$ & ANI & $\begin{array}{l}\text { dDDH } \\
\text { (d0, } \\
\text { in \%) }\end{array}$ & $\begin{array}{l}\text { C.I. } \\
\text { (d0, } \\
\text { in \%) }\end{array}$ & $\begin{array}{l}\mathrm{dDDH} \\
(\mathrm{d} 4, \\
\text { in \%) }\end{array}$ & $\begin{array}{l}\text { C.I. } \\
\text { (d4, } \\
\text { in \%) }\end{array}$ & $\begin{array}{l}\text { dDDH } \\
(\mathrm{d} 6, \\
\text { in \%) }\end{array}$ & $\begin{array}{l}\text { C.I. } \\
\text { (d6, } \\
\text { in \%) }\end{array}$ & $\begin{array}{l}\text { G+C } \\
\text { content } \\
\text { difference } \\
\text { (in \%) }\end{array}$ \\
\hline SC8 & $\begin{array}{l}\text { Streptomyces } \\
\text { bikiniensis } \\
\text { NRRL ISP- } \\
5581\end{array}$ & GCA_000716245 & 88,87 & 34,5 & $\begin{array}{l}{[31.2} \\
- \\
38.1]\end{array}$ & 36 & $\begin{array}{l}{[33.6} \\
-38.6]\end{array}$ & 33,7 & $\begin{array}{l}{[30.7} \\
-36.8]\end{array}$ & 0,83 \\
\hline SC8 & $\begin{array}{l}\text { Streptomyces } \\
\text { cinereoruber } \\
\text { JCM } 4205\end{array}$ & GCA_014649095 & 88,78 & 39,8 & $\begin{array}{l}{[36.4} \\
- \\
43.3]\end{array}$ & 35 & $\begin{array}{l}{[32.5} \\
-37.5]\end{array}$ & 37,9 & $\begin{array}{l}{[35.0} \\
- \\
41.0]\end{array}$ & 0,9 \\
\hline SC8 & $\begin{array}{l}\text { Streptomyces } \\
\text { exfoliatus } \\
\text { NRRL B-2924 }\end{array}$ & GCF_000725545 & 90,72 & 47,5 & $\begin{array}{l}{[44.1} \\
-50.9]\end{array}$ & 40,3 & $\begin{array}{l}{[37.8} \\
- \\
42.8]\end{array}$ & 45,7 & $\begin{array}{l}{[42.7} \\
- \\
48.7]\end{array}$ & 0,21 \\
\hline SC8 & $\begin{array}{l}\text { Streptomyces } \\
\text { gardneri JCM } \\
4375\end{array}$ & GCA_014655085 & 88,87 & 40 & $\begin{array}{l}{[36.7} \\
- \\
43.5]\end{array}$ & 35,5 & $\begin{array}{l}{[33.0} \\
- \\
38.0]\end{array}$ & 38,2 & $\begin{array}{l}{[35.3} \\
- \\
41.3]\end{array}$ & 0,5 \\
\hline SC8 & $\begin{array}{l}\text { Streptomyces } \\
\text { lateritius JCM } \\
4389\end{array}$ & GCA_014649715 & 85,61 & 32,7 & $\begin{array}{l}{[29.4} \\
-36.3]\end{array}$ & 28,9 & $\begin{array}{l}{[26.5} \\
- \\
31.4]\end{array}$ & 30,8 & $\begin{array}{l}{[27.9} \\
- \\
33.9]\end{array}$ & 0,82 \\
\hline SC8 & $\begin{array}{l}\text { Streptomyces } \\
\text { litmocidini } \\
\text { JCM } 4394\end{array}$ & GCA_014649755 & 88,91 & 40,4 & $\begin{array}{l}{[37.0} \\
- \\
43.8]\end{array}$ & 35,8 & $\begin{array}{l}{[33.4} \\
- \\
38.3]\end{array}$ & 38,6 & $\begin{array}{l}{[35.6} \\
- \\
41.6]\end{array}$ & 0,61 \\
\hline SC8 & $\begin{array}{l}\text { Streptomyces } \\
\text { narbonensis } \\
\text { JCM } 4147\end{array}$ & GCA_014649015 & 88,86 & 41,5 & $\begin{array}{l}{[38.2} \\
- \\
45.0]\end{array}$ & 35,2 & $\begin{array}{l}{[32.8} \\
-37.7]\end{array}$ & 39,4 & $\begin{array}{l}{[36.4} \\
- \\
42.4]\end{array}$ & 0,27 \\
\hline SC8 & $\begin{array}{l}\text { Streptomyces } \\
\text { nashvillensis } \\
\text { JCM } 4498\end{array}$ & GCA_014650095 & 89,28 & 41 & $\begin{array}{l}{[37.7} \\
- \\
44.5]\end{array}$ & 36,6 & $\begin{array}{l}{[34.1} \\
- \\
39.1]\end{array}$ & 39,3 & $\begin{array}{l}{[36.4} \\
- \\
42.4]\end{array}$ & 0,12 \\
\hline SC8 & $\begin{array}{l}\text { Streptomyces } \\
\text { omiyaensis } \\
\text { JCM } 4806\end{array}$ & GCA_014650895 & 85,96 & 34,6 & $\begin{array}{l}{[31.2} \\
- \\
38.1]\end{array}$ & 29,4 & $\begin{array}{l}{[27.0} \\
- \\
31.9]\end{array}$ & 32,3 & $\begin{array}{l}{[29.4} \\
- \\
35.4]\end{array}$ & 1,71 \\
\hline SC8 & $\begin{array}{l}\text { Streptomyces } \\
\text { tanashiensis } \\
\text { JCM } 4086\end{array}$ & GCA_014648895 & 89,27 & 40,6 & $\begin{array}{l}{[37.2} \\
- \\
44.0]\end{array}$ & 36,5 & $\begin{array}{l}{[34.1} \\
- \\
39.1]\end{array}$ & 38,9 & $\begin{array}{l}{[36.0} \\
- \\
42.0]\end{array}$ & 0,06 \\
\hline SC8 & $\begin{array}{l}\text { Streptomyces } \\
\text { venezuelae } \\
\text { ATCC } 10712\end{array}$ & GCA_000253235 & 89,68 & 45,7 & $\begin{array}{l}{[42.3} \\
- \\
49.2]\end{array}$ & 37,8 & $\begin{array}{l}{[35.4} \\
- \\
40.4]\end{array}$ & 43,6 & $\begin{array}{l}{[40.6} \\
- \\
46.6]\end{array}$ & 0,32 \\
\hline SC8 & $\begin{array}{l}\text { Streptomyces } \\
\text { wedmorensis } \\
\text { NRRL } 3426\end{array}$ & GCF_000716445 & 88,97 & 39,2 & $\begin{array}{l}{[35.8} \\
- \\
42.7]\end{array}$ & 35,7 & $\begin{array}{l}{[33.3} \\
- \\
38.2]\end{array}$ & 37,6 & $\begin{array}{l}{[34.6} \\
- \\
40.7]\end{array}$ & 0,1 \\
\hline SC8 & $\begin{array}{l}\text { Streptomyces } \\
\text { zaomyceticus } \\
\text { JCM } 4864\end{array}$ & GCA_014656215 & 90,24 & 45,1 & $\begin{array}{l}{[41.8} \\
- \\
48.6]\end{array}$ & 38,9 & $\begin{array}{l}{[36.5} \\
- \\
41.5]\end{array}$ & 43,3 & $\begin{array}{l}{[40.4} \\
- \\
46.4]\end{array}$ & 0,07 \\
\hline SC8 & SC7 & & 99,97 & 99,6 & $\begin{array}{l}{[99.2} \\
- \\
99.8]\end{array}$ & 99,4 & $\begin{array}{l}{[99.0} \\
- \\
99.6]\end{array}$ & 99,8 & $\begin{array}{l}{[99.7} \\
- \\
99.9]\end{array}$ & 0,02 \\
\hline
\end{tabular}

In fact, the results obtained from genomic sequencing of the two actinomycete isolates regarding their taxonomy showed that they belong to:

Group : Terrabacteria

Phylum : Actinobacteria 
Class : Actinobacteria

Order : Streptomycetales

Family : Streptomycetaceae

Genus : Streptomyces

\subsubsection{Phylogenetic analysis}

Phylogenetic analysis based on 16S indicates that the strains cluster with Streptomyces venezuelae ATCC 10712 strain (73\% branch support). On the other side, the whole genome phylogenetic analysis showed that the strains cluster with Streptomyces exofoliatus NRRL B-2924 (100\% branch support). Thus, both phylogeny methods indicate clearly that the two strains are very close and cluster each other (73\% for 16 S and $100 \%$ for WGS) (figures 4 and 5).

\subsubsection{Genome assembly and annotation}

The de novo assembly of the genome, gene prediction and annotation consisted of 1,354 contigs, 7,788,601 bases ( $N 50=$ 9,831; DNA G+C content= 72.19\%), 7,919 coding sequences and 65 sequences encoding tRNA for SC7. However, 1,486 contigs, 7,746,516 bases ( $N 50=8,715$; DNA G+C content= 72.17\%), 7,935 coding sequences and 65 sequences encoding tRNAwere noted for SC8.

The annotation with PATRIC allowed to identify different genes involved in different metabolic pathways including antibiotic biosynthesis, pigment biosynthesis, photosynthesis and degradation of organic compounds. Indeed, in this study, among the thousands products identified, three enzymes involved in the biosynthesis of terpenoid backbone leading to that of terpenoid indole alkaloids including the vincristine and the vinblastine (1-deoxy-D-xylulose 5-phosphate synthase, 1-deoxy-D-xylulose 5phosphate reductoisomerase, 2-C-methyl-D-erythritol 2,4-cyclodiphosphate synthase) were detected for the two actinomycetes isolates genomes (ESM_1 and ESM_2, Online resources).

\section{Discussion}

Isolation of vinblastine and vincristine producing actinomycetes associated to Catharanthus roseus is demonstrated in this work. For fungi and actinomycetes distribution, it was observed that Catharanthus roseus endophytic population was dominated by fungi population while its rhizospheric soil was conquered by actinomycetes population. Among the seven endophytic microorganisms isolated from Catharanthus roseus vegetative organs, one is an actinomycete and the six others are fungi. These results corroborate those found by Caruso et al. (2000) who demonstrated the abundance of the endophytic fungi population isolated from different plants of genus Taxus compared to the endophytic actinomycetes population from the same plants. Dissimilarly to this, ten actinomycetes and two fungi were obtained from Catharanthus roseus rhizospheric soil; this confirms that the soil constitutes the main habitat of actinomycetes (El-Shatoury2006).

Alkaloids test of extracts from microorganisms associated to the plant showed that seven rhizospheric soil actinomycetes strains contained alkaloids. None of the isolated fungi produced the target secondary metabolites.

Alkaloids yield varied from one actinomycete strain to another at a range of 2,3\% to 4,6\%. Compared to alkaloids yield of some medicinal plants such as Emblica officinalis, Acacia catechu, Acacia concina and Hibiscus rosa-sinensis, that of actinomycetes is low. For these plants, alkaloids contents are $11,2 \%, 11,3 \%, 10,2 \%$ and 8,5\%, respectively (Gupta et al. 2013). However, Liu and Liu (2015) reported that the optimization of extraction process is required to improve total alkaloids yield.

Vinblastine and vincristine screening in the positive alkaloid extracts by TLC method showed that two extracts from SC7and SC8 contained respectively vincristine and vinblastine. The two target molecules weren't detected in the most alkaloid 
producing strain (SC6) in this study. The results obtained are different of those obtained by some researchers revealing that endophytic microorganisms isolated from Catharanthus roseus until now, which produced similar secondary metabolites as the plant are fungi. For examples, Fusarium oxysporum isolated from the leaves of the plant (Kumar et al. 2013) and Talaromyces radicus from leaf tissues (Palem et al 2016) produced both vincristine and vinblastine. Besides, Eutypella spp CrP 14 isolated from the stem cuttings of Catharanthus roseus was reported to produce vincristine (Kuriakose et al. 2016), Nigrospora sphaerica from plant leaf was also demonstrated to produce vinblastine (Ayob and Simarani 2016; Ayob et al. 2017) and very early Chaetomium globosum $\mathrm{Cr} 59$ from the stem of the plant produced vinblastine (Zafari et al. 2020).

To our knowledge, this is the first study which demonstrates vinblastine or vincristine producing actinomycetes. Furthermore, it is yet demonstrated that soil rhizospheric actinomycetes can produce analogous secondary metabolites (alkaloids) and the same bioactive molecules (vinblastine and vincristine) than the plant. In our previous study, a similar result was observed; an actinomycete strain isolated from ginger rhizopheric soil (Streptomyces antibioticus) was revealed to produce the same secondary metabolites (terpenes and phenolic compounds) and exhibit similar biological activities (antifungal, antioxidant, anticancer and antimalaria) than those of ginger essential oil (Herivony et al. 2016).

Macroscopic (solid colonies embedded in the agar, presence of colored mycelia, powdery surface) and microscopic (branched vegetative mycelium and aerial mycelium bearing isolated spores) aspects of the two vinblastine and vincristine producing actinomycetes showed that they seemed like the genus Streptomyces (Shriling and Gottlieb 1966).

Confirmation by molecular characterization using whole genome sequencing showed that they belong to the genus Streptomyces. This technique revealed also that ANI and DDH values were strictly below $95 \%$ and $70 \%$, respectively, between the 14 standard genomes used (Streptomyces bikiniensis NRRL B-1049, Streptomyces bikiniensis NRRL ISP-5581, Streptomyces cinereoruber JCM 4205, Streptomyces exfoliates NRRL B-2924, Streptomyces gardneri JCM 4375, Streptomyces lateritius JCM 4389, Streptomyces litmocidini JCM 4394, Streptomyces narbonensis JCM 4147, Streptomyces nashvillensis JCM 4498, Streptomyces omiyaensis JCM 4806, Streptomyces tanashiensis JCM 4086, Streptomyces venezuelae ATCC 10712, Streptomyces wedmorensis NRRL 3426, Streptomyces zaomyceticus JCM 4864) and the two selected strains genomes. However, ANI and DDH values between the two strains (respectively, 99,97\% and 99,6\%) were above the given delineation cutoff. Thus, the two strains were considered as belonging to a same novel Streptomyces species (Richter and Rosselló-Móra 2009; Meier-Kolthoff 2013). In fact, some resemblance of morphological and cultural features of the two isolates cited above could be considered for their belonging to a same new species.

Moreover, metabolic pathways analysis with PATRIC allows to detect three enzymes: 1-deoxy-D-xylulose 5-phosphate synthase encoded by the dxs gene (Chahed et al. 2000), 1-deoxy-D-xylulose 5-phosphate reductoisomerase (dxr gene) and 2C-methyl-D-erythritol 2,4-cyclodiphosphate synthase (mecs gene) (Veau et al. 2000), implied in terpenoid indole alkaloids (TIAs) biosynthesis including the vincristine and the vinblastine in Catharanthus roseus plant. Hence, it would be deduced from these results that the two novel isolated Streptomyces from Catharanthus roseus rhizospheric soil produce the same anticancer metabolites as the plant.

Through the results obtained by TLC for vinblastine and vincristine detection in alkaloids extracts from the two new Streptomyces species isolated from Catharanthus roseus rhizospheric soil and reinforced by those obtained via metabolic pathways analysis of their genome, it would be confirmed that the two novel isolated strains produce vinblastine and vincristine as Catharanthus roseus. However, the biosynthesis mechanism of the two anticancers in the new Streptomyces species should be elucidated.

\section{Conclusion}

From this work, it was revealed that two rhizospheric soil actinomycetes of Catharanthus roseus belonging to a new Streptomyces species produced the same active compounds than the plant (vinblastine and vincristine). To better 
understand the potential of the anticancer from the two novel isolated strains, production parameters optimization, isolation, purification and antiproliferative activity against different cancerous cells will be conducted.

\section{Declarations}

\section{Conflict of interest}

All authors declare that they are no conflict of interest.

\section{Authors contribution}

Each author contributes to the writing of the manuscript according to his/her specialty for each method cited in the text:

- Andriambeloson Herivony Onja, for actinomycetes and fungi isolation from Catharanthus roseus vegetative organs and its rhizospheric soil, conservation of the isolates.

- Andrianantenaina Rigobert, for alkaloids screening of the extracts prepared from the obtained isolates, vinblastine and vincristine screening in the positive alkaloids extracts.

- Rabenandrasana Mamitiana Alain Noah and Collard Jean-Marc, for molecular characterization of the target isolates.

- Ramaroson Luciano, for cultural and morphological characterization of the target isolates.

- Rasolomampianina Rado, for microbial extracts preparation.

The corresponding author compiled the writing from all authors and a rotating reading was done to improve the manuscript. This latter was finalized by the corresponding author.

\section{References}

1. Akshatha VJ, Nalini MS, D' Souza, Prakash HS (2014) Streptomycete endophytes from anti-diabetic medicinal plants of the Western Ghats inhibit alpha-amylase and promote glucose uptake. Lett Appl Microbio/ 58: 433-439. doi: 10.1111/lam.12209

2. Alexopoulos CJ and Mims CW (1979) Introductory Mycology, 3rd ed. Wiley, New York

3. Amna T, Puri SC, Verma V, Sharma JP, Khajuria RK, Musarrat TJ et al (2006) Bioreactor studies on the endophytic fungus Entrophospora infrequens for the production of an anticancer alkaloid camptothecin. Canadian Journal of Microbiology 52: 189-96. doi: 10.1139/w05-122

4. Andriambeloson O, Rasolomampianina R and Raherimandimby M (2014) Selection and characterization of bioactive actinomycetes associated with the medicinal plant Ginger (Zingiber officinale). Journal of International Academic Research for Multidisciplinary 2 (9): 30-45

5. Ayob FW and Simarani K (2016) Endophytic filamentous fungi from a Catharanthus roseus: identification and its hydrolytic enzymes. Saudi Pharmaceutical Journal 24: 273-278. doi: 10.1016/j.jsps.2016.04.019

6. Ayob FW, Simarani K, Abidin NZ and Mohamad J (2017) First report on a novel Nigrospora sphaerica isolated from Catharanthus roseus plant with anticarcinogenic properties. Microbial Biotechnology 10 (4): 926-932. doi:

10.1111/1751-7915.12603

7. Bankevich A, Nurk S, Antipov D, Gurevich AA, Dvorkin M et al (2012) SPAdes: a new genome assembly algorithm and its applications to single-cell sequencing. J Comput Biol 19: 455-477. doi: 10.1089/cmb.2012.0021

8. Bouzidi A, Mahdeb N, Kara N, Benouazah Z (2011) Analyse qualitative et quantitative des alcaloïdes totaux des graines de Datura stramonium L. Agriculture 2 : 79-88 
9. Caruso M, Colombo AL, Fedeli L, Pavesi A, Quaroni S, Saracchi M and Ventrella G (2000) Isolation of endophytic fungi and actinomycetes taxane producers. Ann Microbio/ 50: 3-13

10. Centre de recherche botanique des Kew Gardens, L'état du monde des plantes (2016) Londres

11. Chahed K, Oudin A, Guivarch N, Hamdi S, Chenieux JC, Rideau Metal (2000) 1-Deoxy-D-xylulose 5-phosphate synthase from periwinkle: cDNA identification and induced gene expression in terpenoid indole alkaloid-producing cells. Plant Physiol. Biochem 38: 559-566.doi: 10.1016/S0981-9428(00)00781-6

12. Criscuolo A, Brisse S (2013) Alien Trimmer: a tool to quickly and accurately trim off multiple short contaminant sequences from high throughput sequencing reads. Genomics 102: 500-506. doi: 10.1016/j.ygeno.2013.07.011

13. Cross T (1989) The actinomycetes II- Growth and examination of actinomycetes- some guidelines. In: Williams ST, Sharp ME, Holt JG. Bergey's Manual of Systematic Bacteriology, vol 4. Williams \& Wilkins, Baltimore, pp 2340-2343

14. El-Shatoury S, Abdulla H, El-Karaaly O, El-Kazzaz W and Dewedar A (2006) Bioactivities of endophytic actinomycetes from selected medicinal plants in the world heritage site of Saint Katherine, Egypt. International Journal of Botany 2 (3): 307-312. doi: 10.3923/ijb.2006.307.312

15. Fong HHS, Tin WAM and Farnsworth NR (1977) Phytochemical screening Review. Chicago: University of Illinois, pp 73126

16. Gupta M, Thakur S, Sharma A and Gupta S (2013) Qualitative and Quantitative Analysis of Phytochemicals and Pharmacological Value of Some Dye Yielding Medicinal Plants. Oriental Journal of chemistry 29 (2): 475-481. doi: $10.13005 /$ OJC/290211

17. Hadagali A, Prashanth H, Manasa KH, Chandraprasad M, Pradeep S and Ashok KS (2017) Isolation and detection of vinca alkaloids from endophytes isolated from Catharanthus roseus. European journal of biomedical and pharmaceutical sciences 4(10): 675-683

18. Herivony Onja Andriambeloson, Rado Rasolomampianina, Rahanira Ralambondrahety, Rigobert Andrianantenaina, Marson Raherimandimby, Fidèle Randriamiharisoa (2016) Biological Potentials of Ginger Associated Streptomyces Compared with Ginger Essential Oil. American Journal of Life Sciences 4(6): 152-163. doi: 10.11648/j.ajls.20160406.13

19. Isik K, Chun J, Hah YC and Goodfellow M (1999) Nocardia Salmocida: a fish pathogen. Inter J Syst Bacteriol 49: 833-837

20. James JD, Alice RW, Ramy KA, Thomas B, Ralph B, Rory MB et al (2020) The PATRIC Bioinformatics Resource Center: expanding data and analysis capabilities. Nucleic Acids Research 48 (D1): D606-D612.

https://doi.org/10.1093/nar/gkz943

21. Kumar A, Patil D, Rajamohanan PR, Ahmad A (2013) Isolation, Purification and Characterization of Vinblastine and Vincristine from Endophytic Fungus Fusarium oxysporum Isolated from Catharanthus roseus. Plos one 8 (9): 1- 10. doi: 10.1371/journal.pone.0071805

22. Kuriakose GC, Palem PP, Jayabaskaran C (2016) Fungal vincristine from Eutypella spp-CrP14 isolated from Catharanthus roseus induces apoptosis in human squamous carcinoma cell line-A431. BMC complementary and alternative medicine 16 (1): 302. doi: 10.1186/s12906-016-1299-2

23. Lefort V, Desper R, Gascuel O (2015) FastME 2.0: A comprehensive, accurate, and fast distance-based phylogeny inference program.

Mol Biol Evol 32: 2798-2800. https ://doi.org/10.1093/molbe v/msv15 0

24. Liu Y, Liu C (2015) Extraction Process Optimization of Total Alkaloid from Actinidia arguta.International Conference on Materials, Environmental and Biological Engineering (MEBE). Atlantis Press 131-134

25. Liu Y, Schröder J, Schmidt B (2013) Musket: a multistage k-mer spectrum-based error corrector for Illumina sequence data. Bioinformatics 29: 308-315. doi: 10.1093/bioinformatics/bts690

26. Meier-Kolthoff JP, Auch AF, Klenk HP, Göker M (2013) Genome sequence-based species delimitation with confidence intervals and improved distance functions. BMC Bioinformatics 14: 60.doi: 10.1186/1471-2105-14-60 
27. Meier-Kolthoff JP, Göker M (2019) TYGS is an automated highthroughput platform for state-of-the-art genome-based taxonomy. Nat Commun 10: 2182. https://doi.org/10.1038/s4146 7-019-10210 -3

28. Nguyen MT (2007) Identification des espèces de moisissures, potentiellement productrices de mycotoxines dans le riz commercialisé dans cinq provinces de la région centrale du Vietnam - Étude des conditions pouvant réduire la production des mycotoxines. Thèse de doctorat Université de Toulouse, France

29. Organisation Mondiale de la Santé (2004) Sécurité des patients et protection des plantes médicinales. Centre des Médias, Genève

30. Oskay M, Tamer AU and Azeri C (2004) Antibacterial activity of some actinomycetes isolated from farming soils of Turkey. African Journal of Biotechnology 3 (9): 441-446. doi: 10.5897/AJB2004.000-2087

31. Palem PPC, Kuriakose GC, Jayabaskaran C (2016) An Endophytic Fungus, Talaromyces radicus, Isolated from Catharanthus roseus, Produces Vincristine and Vinblastine, Which Induce Apoptotic Cell Death. Plos one 11 (4): e0153111. https://doi.org/10.1371/journal.pone.0153111

32. Richter M, Rosselló-Móra R (2009) Shifting the genomic gold standard for the prokaryotic species definition. Proc Nat'IAcad Sci U S A 106: 19126-19131. https://doi.org/10.1073/pnas.0906412106

33. Rodriguez LMR, Konstantinidis KT (2016) The enveomics collection: a toolbox for specialized analyses of microbial genomes and metagenomes. Peer J Preprints 4:e1900v1. https://doi.org/10.7287/peerj.preprints.1900v1

34. Shriling EB and Gottlieb D (1966) Methods for characterization of Streptomyces species. Int. J Syst Bact 16: 313 340.doi: 10.1099/00207713-16-3-313

35. Shweta S, Zuehlke S, Ramesha BT, Priti V, Mohana Kumar P, Ravikanth G et al(2010) Endophytic fungal strains of Fusariumsolani, from Apodytes dimidiata E. Mey. Ex Arn (Icacinaceae) produce camptothecin, 10-hydroxycamptothecin and 9-methoxycamptothecin. Phytochemistry71: 117-22. doi: 10.1016/j.phytochem.2009.09.030

36. Veau B, Courtois M, Oudin A, Chenieux JC, Rideau M, Clastre M (2000) Cloning and expression of cDNAs encoding two enzymes of the MEP pathway in Catharanthus roseus. Biochim Biophys Acta 1517: 159-163.doi: 10.1016/s01674781(00)00240-2

37. Zafari D, Leylaie S, Tajick MA (2020) Isolation and identification of vinblastine from the fungus of Chaetomium globosum Cr95 isolated from Catharanthus roseus plant. Biological journal of microorganism 8 (32): 1-14. doi: 10.22108/BJM.2018.107927.1095

38. Zhao J, Li C, Wang W, Zhao C, Luo M, Mu F et al (2013) Hypocrealixii, novel endophytic fungi producing anticancer agent cajanol, isolated from pigeon pea (Cajanuscajan [L] Mill sp. J Appl Microb 115: 102-113. doi: 10.1111/jam.12195

\section{Figures}




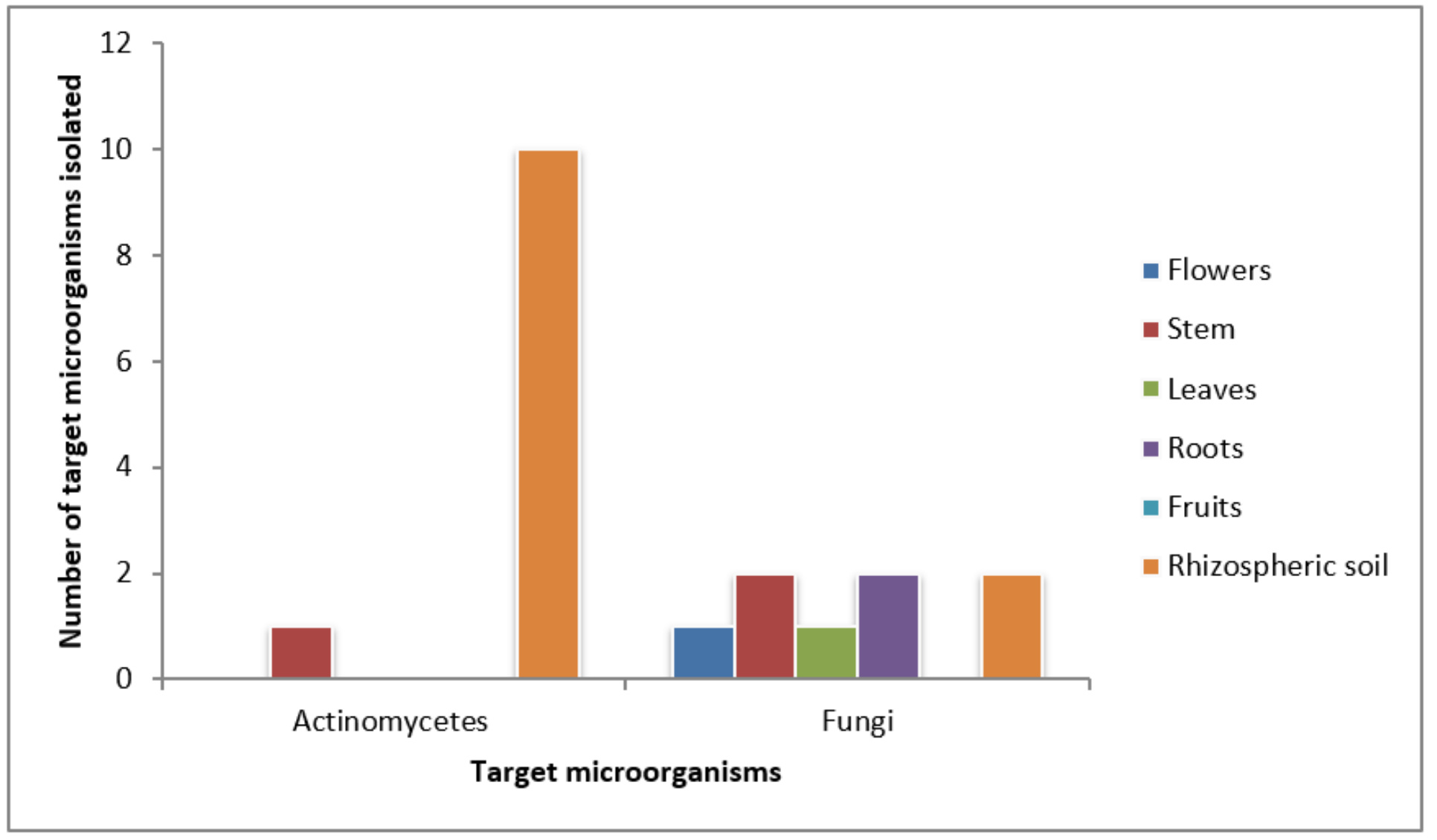

\section{Figure 1}

Number of actinomycetes and fungal strains isolated from Catharanthus roseus plant and its rhizospheric soil

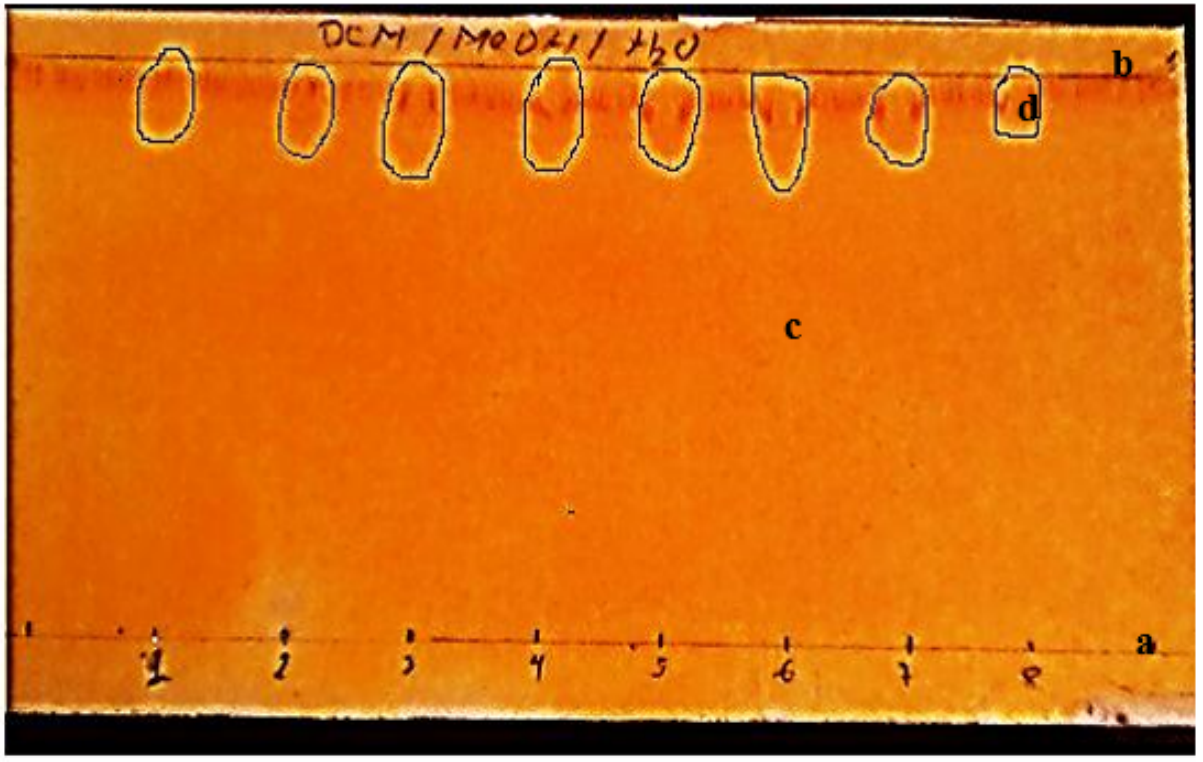

\section{Figure 2}

Chromatographic profile for vinblastine and vincristine screening in alkaloid extracts from Catharanthus roseus rhizospheric soil 1 : vinblastine; 2 : vincristine; 3 : alkaloids from the isolate SC $; 4$ : alkaloids from the isolate SC4; 5 : alkaloids from the isolate SC5 6 : alkaloids from the isolate SC6, 7 : alkaloids from the isolate SC7; 8 : alkaloids from the isolate SC8 a: deposit line; b: front line; c: plate saturated with Dragendorff's reagent; d: alkaloids migration 

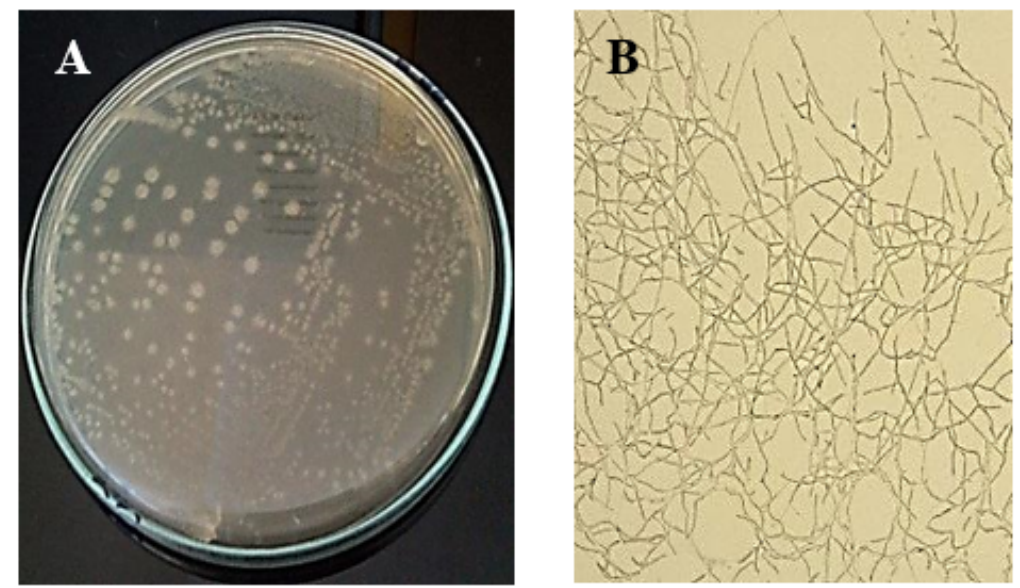

Isolate SC7
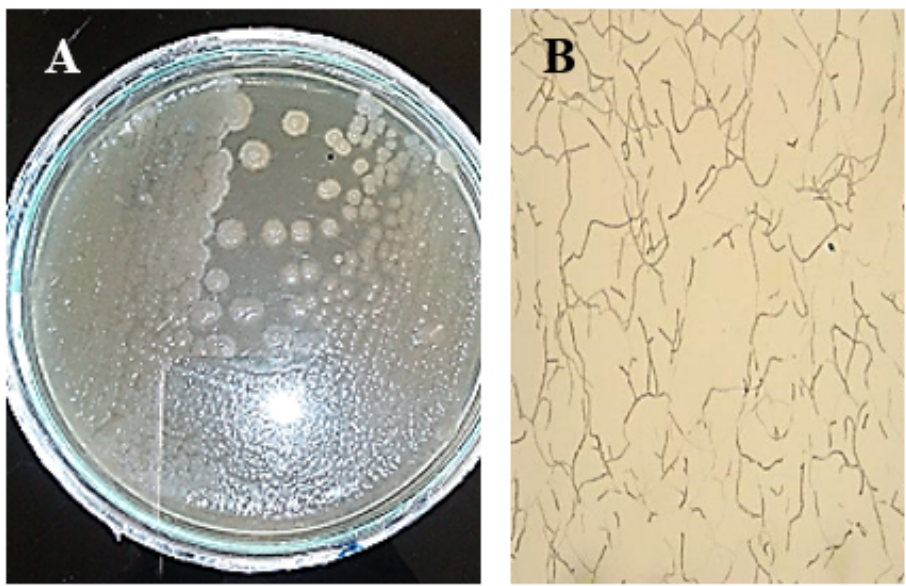

Isolate SC8

\section{Figure 3}

Macroscopic and microscopic aspects of vinblastine and vincristine producing actinomycetes isolated from Catharanthus roseus rhizospheric soil A: cultural characteristics on SCA medium; B: morphological characteristics by microscopic observation (LEICA x 40)

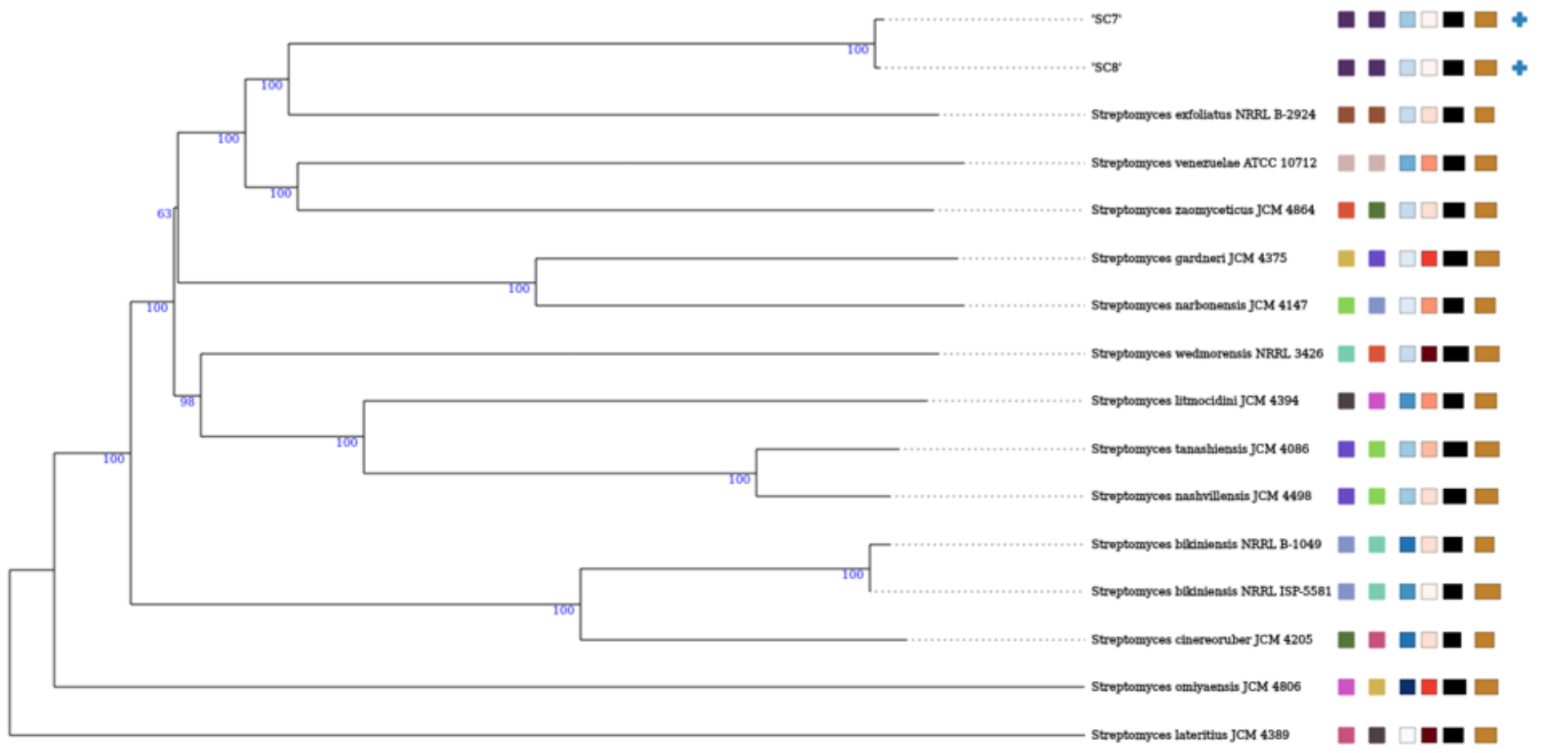

\section{Figure 4}

Phylogenomic tree of the isolates SC7 and SC8 based on genome sequences in the TYGS tree inferred with FastME 2.1.6.1 


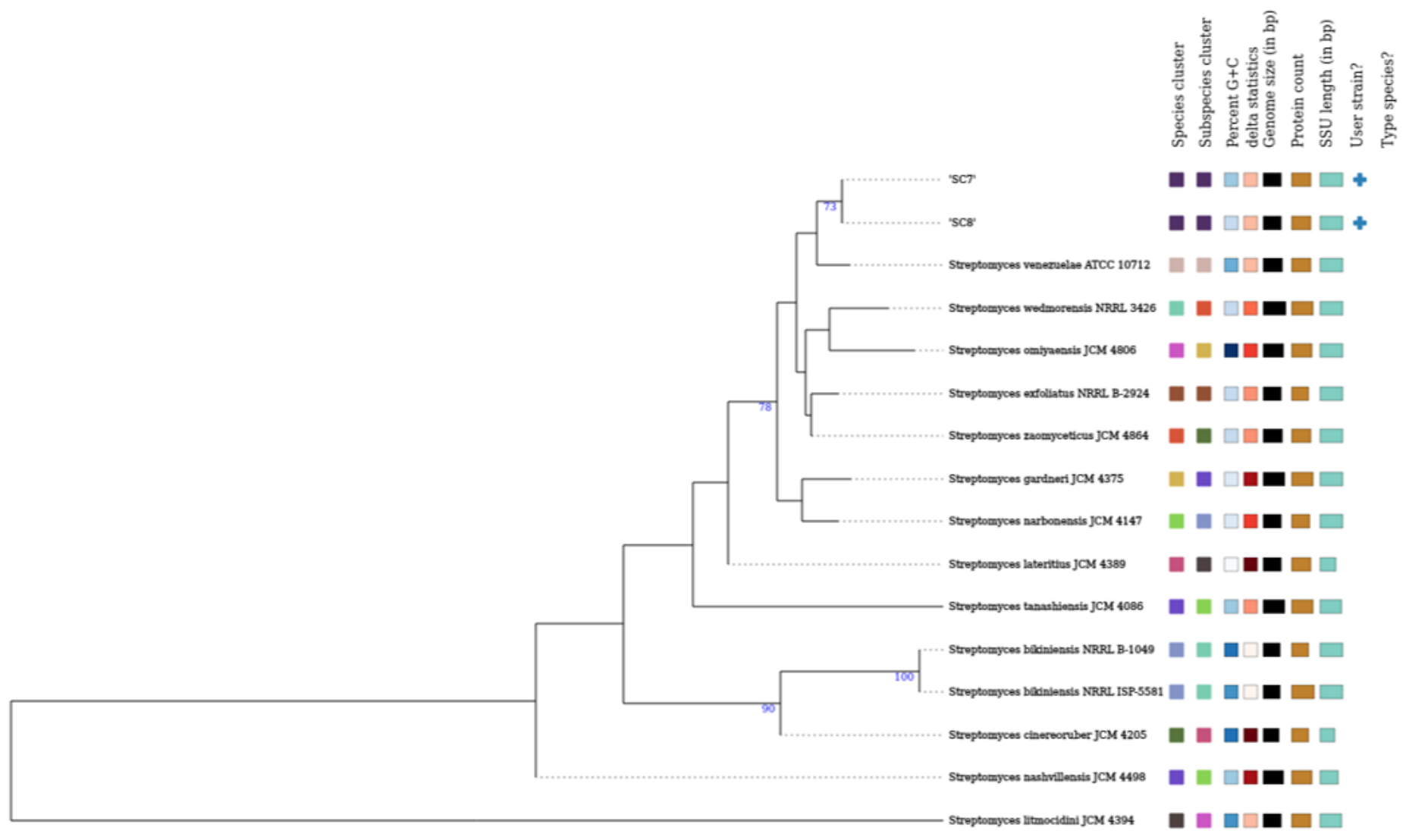

Figure 5

16S phylogenetic tree of the isolates SC7 and SC8 based on genome sequences in the TYGS tree inferred with FastME 2.1.6.1

\section{Supplementary Files}

This is a list of supplementary files associated with this preprint. Click to download.

- ESM1.xIsx.xIsx

- ESM2.xlsx.xIsx 